\title{
Common FTO rs9939609 variant and risk of type 2 diabetes in Palestine
}

\author{
Anas Sabarneh $^{1 \dagger}$, Suheir Ereqat ${ }^{1,7^{*}+}$ C, Stéphane Cauchi ${ }^{2,3,4,5}$, Omar AbuShamma ${ }^{1}$, Mohammad Abdelhafez ${ }^{1}$, \\ Murad Ibrahim ${ }^{6}$ and Abdelmajeed Nasereddin ${ }^{7}$
}

\begin{abstract}
Background: Genetic and environmental factors play a crucial role in the development of type 2 diabetes mellitus (T2DM) and obesity. This study aimed to investigate the association of the fat-mass and obesity-associated gene (FTO) rs9939609 variant with T2DM and body mass index (BMI) among Palestinian population.

Methods: A total of 399 subjects were recruited, of whom 281 were type 2 diabetic patients and 118 normoglycemic subjects. All of them were unrelated, aged $>40$ years and recruited within the period 2016-2017. The A allele of FTO rs9939609 was identified by PCR-RFLP.

Results: Significant association of the minor allele A of FTO rs9939609 and T2DM risk was observed with an allelic odd ratio of 1.92 (95\% Cl [1.09-3.29], $p=0.02$ ) adjusted for age and gender, this association partly attenuated when adjusted for BMI with OR of 1.84, (95\% $\mathrm{Cl}[1.04-3.05], p=0.03)$. Stratified data by glycemic status across FTO genotypes showed that A allele was marginally associated with increased BMI among diabetic group $(p=0.057)$ but not in control group $(p=0.7)$. Moreover, no significant association was observed between FTO genotypes and covariates of age, gender, T2DM complications or any tested metabolic trait in both diabetic and nondiabetic individuals $(p>0.05)$.

Conclusions: The variant rs9939609 of the FTO gene was associated with T2DM in Palestine. This is the first study conducted on this gene in the Palestinian population and provides valuable information for comparison with other ethnic groups. Further analysis with larger sample size is required to elucidate the role of this variant on the predisposition to increased BMI in Palestinians.
\end{abstract}

Keywords: FTO, rs9939609 variant, T2DM, BMI, Palestine

\section{Background}

Type 2 diabetes mellitus (T2DM) is the most common type of diabetes as it accounts for more than $90 \%$ of all diabetes cases worldwide (World Health Organization) [1]. Polymorphisms within the fat-mass and obesity-associated gene (FTO) are of particular interest as they have known effect on obesity which is a major risk factor for T2DM. A genome-wide association study (GWAS) conducted in 2007, confirmed that rs9939609 variant located within the first intron of the FTO gene predisposes European

\footnotetext{
* Correspondence: sereqat@staff.alquds.edu

${ }^{\dagger}$ Anas Sabarneh and Suheir Ereqat contributed equally to this work.

${ }^{1}$ Biochemistry and Molecular Biology Department, Faculty of Medicine,

Al-Quds University, Abu Dis-East Jerusalem, Palestine

${ }^{7}$ Al-Quds Nutrition and Health Research Institute - Faculty of Medicine,

Al-Quds University-Palestine, Abu Dis-Jerusalem, Palestine

Full list of author information is available at the end of the article
}

populations to diabetes through an effect on body mass index (BMI) [2, 3], while other reports from South Asian population showed that $F T O$ gene variants increase the risk of type 2 diabetes independent of BMI [4]. Since then, several studies represent various ethnic populations, confirmed strong associations of the FTO rs9939609 with obesity $[5,6]$. This association was not replicated in the Chinese Han population and African Americans [7, 8]. It is well-established that dyslipidemia is a risk factor for cardiovascular diseases (CVD) in diabetic patients. However, a study conducted by Doney et al. [9] demonstrated that A allele rs9939609 in the FTO gene increases the risk of myocardial infarction in patients with T2DM independent of BMI, glycated hemoglobin, mean arterial pressure and dyslipidemia. Moreover, a significant association of FTO variant

(c) The Author(s). 2018 Open Access This article is distributed under the terms of the Creative Commons Attribution 4.0 International License (http://creativecommons.org/licenses/by/4.0/), which permits unrestricted use, distribution, and reproduction in any medium, provided you give appropriate credit to the original author(s) and the source, provide a link to the Creative Commons license, and indicate if changes were made. The Creative Commons Public Domain Dedication waiver (http://creativecommons.org/publicdomain/zero/1.0/) applies to the data made available in this article, unless otherwise stated. 
was found in Indian patients with T2DM without dyslipidemia [10].

A sex-specific effect of $F T O$ variants on susceptibility to obesity have been shown, a study -in 2016- indicated that the effect of FTO variants on T2DM susceptibility in Japanese men but not women is mediated through FTO effect on BMI [11]. In 2018, a case control study conducted on obese Iranian women showed that several FTO variants including rs9939609 were associated with T2DM and obesity as well [12]. A recent spatial and meta-analysis suggested a region-related associations between FTO rs9939609 and T2DM [13]. Thus, the reported results were not consistent in different ethnic population.

In Palestine, the prevalence of DM (for adults aged > 25 years) was $15.3 \%$ in 2010 , but estimates have placed to be as high as $20.8 \%$ by $2020[14,15]$. Diabetes and its complications are estimated to account for approximately $5.7 \%$ of all deaths in Palestine [16]. The prevalence of overweight and obesity is rapidly escalating in the youth and adults, probably due changes in lifestyle, further enhancing the risk of diabetes. In 2016, a cross sectional study among the students at An-Najah National University in Nablus district (North Palestine) showed that the prevalence of overweight and obesity was $26.2 \%$, with significant increase in males (36.4\%) compared to females (19.1\%) [17]. Genetic association studies of T2DM among Palestinians are scarce. Two studies conducted by Ereqat and colleagues in 2009 [18, 19] investigated the genetic association of Pro12Ala Polymorphism of the PPAR-Gamma 2 gene and rs7903146 variant in the transcription factor 7 like 2 gene (TCF7L2) with T2DM. However, no studies have been conducted to determine the genetic association of FTO variants with T2DM and/ or obesity. Therefore, our study aimed to examine the association between the FTO rs9939609 SNP with the risk of T2DM and its-related phenotypes in Palestinian population.

\section{Methods}

\section{Study population}

A total of 399 unrelated individuals were recruited from different cities in Palestine. Two-hundred eighty one cases, aged $>40$ years, were diagnosed by T2DM according to WHO criteria based on fasting plasma glucose $126 \mathrm{mg} / \mathrm{dl}$ and/or currently being treated with medication for diabetes. All participants were recruited within the period of 2016-2017 in collaboration with UNRWA clinics (Hebron and Ramallah, Palestine). The anthropometric measurements were collected from their medical records that included age, sex, family history, drug history, medical history and other related information.

Fasting blood was collected for biochemical tests and DNA studies. All the cases with probable diagnosis of type 1 diabetes were excluded. The control group $(n=$ 118), was selected from individuals who came to the same clinic for health check-up with no past medical history for T2DM and no family history of diabetes in first-degree relations. Age at examination was $>40$ years.

\section{Biochemical testing, DNA extraction and genotyping}

Five milliliters of blood were obtained after overnight fast, collected in EDTA tubes, centrifuged at room temperature. Plasma glucose, cholesterol, HDL cholesterol, and triglyceride were determined by enzymatic methods as described by manufacturer's instructions (Human, Wiesbaden, Germany). Genomic DNA was extracted from whole blood $(300 \mu \mathrm{l})$ using genomic DNA purification kit QIAamp according to the manufacturer instructions (Qiagen, Hilden, Germany). DNA samples were stored at $4{ }^{\circ} \mathrm{C}$ for further analyses. Genotyping of the FTO rs9939609 SNP was done by PCR-based restriction fragment length polymorphism (RFLP) analysis as previously described [20] with the following modifications. The PCR reactions were carried out using $20 \mathrm{ng}$ of purified genomic DNA samples, with $0.4 \mu \mathrm{M}$ of the forward and reverse primers using PCR-Ready Supreme mix (Syntezza Bioscience, Jerusalem) in a final volume of $25 \mu$ l. The genotypes patterns were determined by $2 \%$ agarose gel electrophoresis (Agarose; Sigma-Aldrich, Munich, Germany) stained with Ethiduim bromide. A $5 \%$ masked, random sample of cases and controls were re-amplified and sent for sequencing to confirm the genotyping method.

\section{Statistical analysis}

The genotype frequencies were tested for Hardy-Weinberg equilibrium using a chi-square test through the website http://www.oege.org/software/hwe-mr-calc.html. All statistical analysis was performed using SPSS v23.0 (SPSS, Chicago, IL). Pearson's Chi-square test was used to compare allelic and genotypic frequencies between the diabetic

Table 1 Clinical and biochemical characteristics of case and control groups

\begin{tabular}{llll}
\hline & cases $(n=281)$ & controls $(n=118)$ & \\
Parameters & & & P-value \\
\hline M:F ratio & $94: 187$ & $48: 70$ & \\
Age at diagnosis (years) & $50.39+11.04$ & $\mathrm{NA}$ & \\
Age at sampling (years) & $58.15 \pm 12.09$ & $49.62 \pm 8.71$ & $0.0001^{*}$ \\
BMl & $32.9 \pm 6.6$ & $29.2 \pm 6.2$ & $<0.0001^{*}$ \\
SBP (mmHg) & $136.21 \pm 17.42$ & $122.33 \pm 11.44$ & $0.0001^{*}$ \\
DBP (mmHg) & $80.21 \pm 10.83$ & $76.72 \pm 9.37$ & $0.0024^{*}$ \\
FBS (mg/dl) & $163.31 \pm 58.72$ & $87.14 \pm 7.82$ & $0.0001^{*}$ \\
TC (mg/dl) & $187.91 \pm 47.53$ & $182.15 \pm 40.95$ & 0.251 \\
Treatment (OHA,C,l)(n) & $204,71,6$ & $\mathrm{NA}$ & \\
\hline
\end{tabular}

Values are expressed as means $\pm \mathrm{SD},{ }^{*} P<0.05$ is considered to be significant $N A$ Not applicable. Treatment at time of recruitment $\mathrm{OHA}$ oral hypoglycemic agent, $C$ combination of insulin and hypoglycemic agent, I insulin 

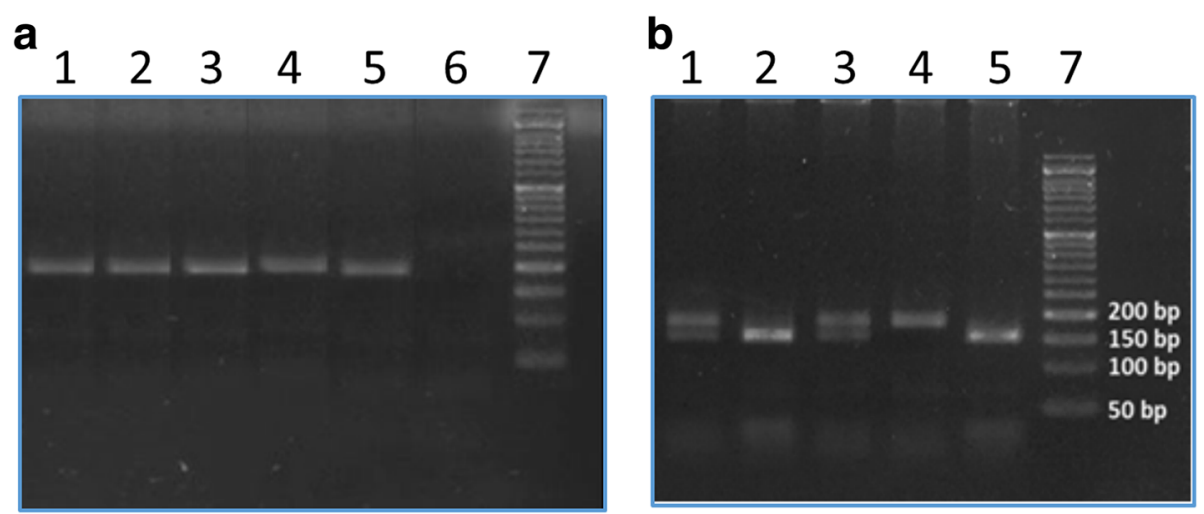

Fig. 1 Agarose gel electrophoresis (2\%) of FTO gene variant (a) PCR products showing 187 bp amplicon (Lanes 1-5), lane 6: Negative control, lane 7: 50 bp ladder. (b) Digested PCR product representing different genotypes: Lanes 1, 3: AT genotype; Lanes 2, 5: AA genotype; Lane 4: Tा genotype; Lane 7: 50 bp ladder

and nondiabetic groups. ANOVA was used to assess the association between FTO genotypes and continuous variables. Logistic regression by $\mathrm{R}$ statistics (V 3.4.4) software was used to measure odd ratio (OR) for T2DM adjusted for age, gender and BMI.

\section{Results}

\section{Biochemical characteristics of the study participants}

The biochemical and anthropometric results of the 281 T2DM patients and 118 nondiabetic subjects are shown in Table 1. As expected, significant differences in biochemical parameters was observed between the two groups $(p<0.05)$. However, the mean total cholesterol was not significant between diabetic and non diabetic groups $(p=0.25)$. Among T2DM group, $64.4 \%(n=181)$ were obese (BMI $>30 \mathrm{~kg} / \mathrm{m} 2), 30.2 \%(n=85)$ were overweight $(25-29.9 \mathrm{~kg} / \mathrm{m} 2)$ and $5.3 \%(n=15)$ were nonobese $(\mathrm{BMI}<30 \mathrm{~kg} / \mathrm{m} 2)$. Of them, $76.6 \%$ were treated with oral hypoglycemic agent, $25.3 \%$ received a combination of insulin and oral hypoglycemic agents and $2.1 \%$ were treated with insulin. Of these patients, 14.6, 12.1, 7.8 and $6 \%$ had cardio vascular disease (CVD), nephropathy, diabetic foot and retinopathy, respectively. Noteworthy, 75\% of the cases had T2DM first-degree relatives. Among the control group, $44.1 \%(n=52)$ were obese, $22.9 \%(n=27)$ were overweight and $33.1 \%(n=39)$ were not obese.

\section{Analysis of FTO variant}

FTO genotyping (rs9939609) was performed by PCR followed by RFLP. The presence of product was verified on a $2 \%$ agarose gel stained with ethidium bromide, a band of 187 bp was observed as shown in Fig. 1a. The PCR product was digested by ScaI restriction enzyme and visualized by $2 \%$ agarose gel. A band of 187 bp was observed for the TT genotype, two bands of 154, 33 bp was observed for the AA genotype while three bands of 187 ,
154, 33 bp were observed for the heterozygous genotype AT as shown in Fig. 1b.

\section{Association of FTO variant and T2DM}

The genotype and allele frequency of FTO gene polymorphism (rs9939609) a;mong the two groups were analyzed and compared as shown in Table 2. Our results revealed that carriers of AA genotype was significantly higher in T2DM subjects compared to non diabetic individuals $(36 \%)$ vs $(16 \%))(p=0.003)$. The genotyping distribution was in line of Hardy Weinberg equilibrium in all cases and controls $(p=>0.05)$. Logistic regression analysis was performed for AT and AA genotypes with TT as a reference genotype. We found that the AT genotype conferred 2.1 times higher risk for T2DM compared to TT genotypes unadjusted $p<0.0001$ (Table 3). As our controls were younger than diabetic cases, logistic regression model adjusted for age and gender was used, and showed that allelic odd ratio was 1.92 (95\% CI [1.09-3.29], $p=0.02)$. This association remained significant even after adjusting for age, gender and BMI (OR 1.84, 95\%CI (1.04-3.05)). The highest risk was observed among AA carriers compared to those with TT genotypes (OR 4.03, 95\% CI $(2.01-8.06) p<0.0001)$ as shown in Table 3.

Table 2 Allelic and genotypic frequency of FTO variant (rs9939609) among T2DM cases and controls

\begin{tabular}{llll}
\hline Genotype & cases $(n)$ & Controls $(n)$ & All Subjects $(n)$ \\
\hline$\pi T$ & 51 & 45 & 96 \\
AT & 129 & 54 & 183 \\
AA & 101 & 19 & 120 \\
A allele (\%) & 58.8 & 38.9 & 53 \\
\hline
\end{tabular}


Table 3 Association of FTO variant (rs9939609) with T2DM

\begin{tabular}{lllll}
\hline Genotype & $\mathrm{OR}(95 \% \mathrm{Cl})$ & $P$-value & $\mathrm{OR}(95 \% \mathrm{Cl})$ & ${ }^{*} P$-value \\
\hline AA vs TT & $4.69(2.49-8.83)$ & $<0.0001$ & $4.03(2.01-8.06)$ & $<0.0001^{*}$ \\
AT vs TT & $2.11(1.26-3.52)$ & $<0.0001$ & $1.84(1.04-3.05)$ & $0.034^{*}$ \\
AA vs & & & & \\
(AT+TT) & $2.78(1.72-4.49)$ & $<0.0001$ & $2.71(1.5-4.9)$ & $0.001^{*}$ \\
\hline
\end{tabular}

* $P$ values were from logistic regression models adjusted for age, gender and $\mathrm{BMI}, P<0.05$ was considered significant

\section{Association of FTO variant with BMI}

The entire data including all the study subjects $(n=399)$ was stratified based on FTO genotypes, a significant association was found between FTO genotypes and mean BMI, the AA genotypes had the highest BMI $(33.29 \pm$ 7.2 ), unadjusted $p=0.03$. Because of potential confounding between T2DM and increased BMI as proxy measure of obesity, the data was stratified by glycemic status across FTO genotypes. Among diabetic group, a trend of increasing mean BMI was observed among the three genotypes: AA carriers had the highest BMI $(34.11 \pm 7.1)$ compared to AT $(32.32 \pm 6.1)$ and TT carriers $(31.86 \pm$ $6.5)$ but it was not significant $(p=0.057)$. However, this increasing trend was not found among the control group $(p=0.7)$ as shown in Table 4. Furthermore, no association was found between the FTO genotype and gender, age, plasma total cholesterol, as well as systolic and diastolic blood pressure among the two groups. Among diabetic group, no association was found between the FTO genotype and cardio vascular disease or diabetes complications $(p>0.05)$.

\section{Discussion}

To our knowledge, this study is the first to investigate the association of the FTO variant rs9939609 with type 2 diabetes and BMI in Palestine. The significance of common variants in the FTO gene for susceptibility to adiposity have been highlighted by large-scale studies among Europeans while conflicting results were reported in Asian populations [21, 22]. Our study showed a significant association of FTO variant rs9939609 with
T2DM after adjustment by age and gender with an allelic odds ratio of 1.92 (95\% CI [1.09-3.29], $p=0.02$ ). Moreover, we noted that the association of FTO variant rs9939609 with T2DM was partially attenuated by adjusting for BMI with odd ratio of 1.84, 95\%CI (1.043.05) $p=0.03$, suggesting that the FTO -T2DM association was not completely mediated through $F T O$ variant effect on BMI. Similar results were found in Indian, American and Chinese populations [4, 10, 23]. Vasan and colleagues [24] provided evidence that FTO-T2DM risk -among Asian Indians- was attenuated but not fully abolished when adjusting to BMI. In contrast, a recent study conducted in Kuwaiti population did not observe an association between the FTO rs9939609 with T2DM risk [25]. Two studies in North Indians and Asian Indians demonstrated a strong association of FTO rs9939609 with T2DM independent of BMI $[4,26]$. However, contradictory results for association of FTO variants with T2DM have been reported in different ethnic groups of India [27]. Furthermore, a meta-analysis study conducted in South Asia showed that BMI and central obesity can partly account for the association of A allele of the FTO gene and diabetes, whereas this association was much reduced when adjusted for BMI in Europeans indicating ethnic-specific associations [28]. On the other hand, several studies revealed a strong association between different variants within the FTO gene and BMI or diabetes supporting that the impact of FTO on obesity or diabetes is population-dependent [29-32]. A recent study conducted by Wang and colleagues [33] showed that FTO protein expression in T2DM patients was higher than in healthy controls which was positively correlated with T2DM severity, BMI and waist circumference.

Our study revealed that, among all study subjects, the TT carriers had lower BMI compared to AT and AA carriers (unadjusted $p=0.03$ ) but when the mean BMI was stratified by glycemic status across FTO genotypes, the association with BMI was lost in the control group $(p=0.7)$. In diabetic group, an additive trend of the allele A with increased BMI was observed but was not significant $(p=0.057)$. However, further studies with larger sample size and greater statistical

Table 4 Mean trait values stratified by glycemic status across FTO genotypes

\begin{tabular}{|c|c|c|c|c|c|c|c|c|}
\hline & \multicolumn{4}{|c|}{ T2DM $(n=281)$} & \multicolumn{4}{|c|}{ Control $(n=118)$} \\
\hline & $\overline{A A}$ & AT & $\pi$ & ${ }^{*} P$-value & $\mathrm{AA}$ & AT & $\pi$ & ${ }^{*} P$-value \\
\hline Number & 101 & 129 & 51 & & 19 & 54 & 45 & \\
\hline BMI (kg/m2) & $34.11 \pm 7.1$ & $32.32 \pm 6.1$ & $31.86 \pm 6.5$ & 0.057 & $28.89 \pm 6.96$ & $29.77 \pm 6.1$ & $28.75 \pm 5.8$ & 0.688 \\
\hline SBP (mmHg) & $133.94 \pm 16.9$ & $138.26 \pm 16.4$ & $135.47 \pm 20.4$ & 0.166 & $119.3 \pm 16.3$ & $124.2 \pm 10.7$ & $121.4 \pm 9.5$ & 0.222 \\
\hline $\mathrm{DBP}(\mathrm{mmHg})$ & $78.68 \pm 11.9$ & $80.94 \pm 10.1$ & $81.35 \pm 10.5$ & 0.206 & $72.8 \pm 9.2$ & $76.7 \pm 10.2$ & $78.5 \pm 8.1$ & 0.087 \\
\hline FBS (mg/dl) & $159.11 \pm 54.3$ & $163.97 \pm 58.9$ & $169.94 \pm 65.6$ & 0.553 & $85.9 \pm 6.9$ & $87.7 \pm 7.4$ & $86.9 \pm 8.8$ & 0.693 \\
\hline TC (mg/dl) & $189.16 \pm 49.3$ & $188.57 \pm 49.2$ & $183.71 \pm 39.8$ & 0.783 & $168.1 \pm 40.1$ & $188.5 \pm 48.8$ & $180.4 \pm 28.2$ & 0.165 \\
\hline
\end{tabular}

${ }^{*} P<0.05$ was considered significant, obtained by ANOVA. Values are expressed as means $\pm \mathrm{SD}$. Bold number showed marginally significant association between $\mathrm{A}$ allele and increased BMI among diabetic group 
power are needed to replicate these findings. In 2016, a study conducted on Egyptian children and adolescents didn't show an association between the polymorphism rs9939609 and BMI. However, that study revealed a significant correlation between LDL and FTO rs9939609 supporting the idea that this variant can be a determinant of obesity due to its effect on the lipid profile [34].

The high prevalence of obesity among our diabetic and control group (64 and $44 \%$, respectively) could be attributed to other variants within $F T O$ and or other genes which can be modulated by environmental factors and lifestyle. Anyhow, as our diabetic cases were older and had higher BMI than controls, we adjusted for the possible confounding effect of age, sex, and BMI in all the logistic regression analyses while investigating T2DM risk across FTO genotypes. Recently, a study conducted by Celis-Morales et al. [35] reported that physical activity attenuates the effect of FTO on BMI. Another study conducted on Emirati people showed that the AA carriers who were physically active had a lower mean BMI than those who were physically inactive, while other studies conducted on African Americans and Europeans showed no such interaction [36, 37]. Furthermore, a recent cross sectional study in a multiethnic population suggested that high dietary protein intake may protect against the effects of risk variants in the FTO gene on BMI and waist circumference [28]. In this study and due to the lack of data regarding physical activity or diet intake, we were unable to examine the influence of physical activity / diet on the impact of FTO variant on BMI. Although FTO -T2DM association was found, the lack of association between FTO rs9939609 and obesity is most probably due to the small sample size -and thereby decreased statistical power- which was the most important limitation in this study and thus larger sample size is required to verify these results. However, weight, skinfold thicknesses, body fat percentage and waist circumference are reported to be more reliable markers of obesity than BMI [38]. We believe that obesity-related genetic variants also modulate glucose-insulin secretion. Therefore, leaner cases should be recruited while investigating gene-T2DM association among Palestinians.

On the other hand, we did not find any association of FTO rs9939609 with the T2DM complications and the prevalence of CVD among the studied population. This is consistent with recent findings showing no association of FTO rs9939609 variant with diabetic retinopathy and nephropathy [39]. However, a meta-analysis study reported significant association of the FTO rs9939609 variant with CVD risk, which was independent of BMI and other conventional CVD risk factors [40].

\section{Conclusion}

The FTO rs9939609 variant was significantly associated with T2DM in Palestine. However, further analysis with larger sample size and data on physical activity and diet intake is required to elucidate the role of this variant and other variants of FTO gene on the predisposition to increased BMI in Palestinians.

\section{Abbreviations}

BMI: Body max index; CVD: Cardiovascular disease; FTO: Fat-mass and obesity-associated gene; GWAS: Genome-wide association; OR: Odd ratio; PCR: Polymerase chain reaction; RFLP: Restriction fragment length polymorphism; T2DM: Type 2 diabetes mellitus; TCF7L2: Transcription factor 7 like 2; WHO: World Health Organization

\section{Acknowledgments}

The authors gratefully acknowledge the UNRWA outpatient clinic members (Hebron and Ramallah, Palestine) who contributed to the patients' recruitment, and all subjects who participated to this study.

\section{Funding}

The deanship of scientific research-Al-Quds University provided the financial support.

\section{Availability of data and materials}

The data sets used and /or analyzed during the current study are available from the corresponding author upon reasonable request.

\section{Authors' contributions}

SE and AS performed the experiments and wrote the manuscript; SE and SC analyzed the data; AS, OA and MA collected the samples; AN and MI performed sequencing and analysis; SE, SC and AN designed and supervised the experiments, reviewed and revised the manuscript. All authors read and approved the final manuscript.

Ethics approval and consent to participate

All the study procedures were approved by the Ethics Committee of Al-Quds University (Rf no. 2/SRC/4).

Informed consent was obtained from all individual participants included in the study.

Consent for publication

Written consent was obtained from all participants to publish their data.

Competing interests

The authors declare that they have no competing interests.

\section{Publisher's Note}

Springer Nature remains neutral with regard to jurisdictional claims in published maps and institutional affiliations.

\section{Author details}

${ }^{1}$ Biochemistry and Molecular Biology Department, Faculty of Medicine, Al-Quds University, Abu Dis-East Jerusalem, Palestine. ${ }^{2}$ CNRS, UMR8204, Lille, France. ${ }^{3}$ INSERM, U1019, Lille, France. ${ }^{4}$ Université de Lille, Lille, France. IInstitut Pasteur de Lille, Centre d'Infection et d'Immunité de Lille, Lille, France. ${ }^{6}$ Microbiology and immunology Department-Faculty of Medicine, Al-Quds University-Palestine, Abu Dis-East Jerusalem, Palestine. ${ }^{7}$ Al-Quds Nutrition and Health Research Institute - Faculty of Medicine, Al-Quds University-Palestine, Abu Dis-Jerusalem, Palestine.

Received: 1 May 2018 Accepted: 17 August 2018

Published online: 31 August 2018

\section{References}

1. World Health Organization. WHO Media centre. http://www.who.int/ mediacentre/factsheets/fs138/en/. Accessed 14 Aug 2018.

2. Frayling TM, Timpson NJ, Weedon MN, Zeggini E, Freathy RM, Lindgren CM, et al. A common variant in the FTO gene is associated with body mass index and predisposes to childhood and adult obesity. Science. 2007:316: 889-94. 
3. Scuteri A, Sanna S, Chen WM, Uda M, Albai G, Strait J, et al. Genome-wide association scan shows genetic variants in the FTO gene are associated with obesity-related traits. PLoS Genet. 2007;3:e115.

4. Sanghera DK, Ortega L, Han S, Singh J, Ralhan SK, Wander GS, et al. Impact of nine common type 2 diabetes risk polymorphisms in Asian Indian Sikhs: PPARG2 (Pro12Ala), IGF2BP2, TCF7L2 and FTO variants confer a significant risk. BMC Med Genet. 2008;9:59.

5. Cecil JE, Tavendale R, Watt P, Hetherington MM, Palmer CN. An obesityassociated FTO gene variant and increased energy intake in children. $\mathrm{N}$ Engl J Med. 2008:359:2558-66.

6. Berentzen T, Kring SI, Holst C, Zimmermann E, Jess T, Hansen T, et al. Lack of association of fatness-related FTO gene variants with energy expenditure or physical activity. J Clin Endocrinol Metab. 2008;93:2904-8.

7. Li H, Wu Y, Loos RJ, Hu FB, Liu Y, Wang J, et al. Variants in the fat mass- and obesity-associated (FTO) gene are not associated with obesity in a Chinese Han population. Diabetes. 2008;57:264-8.

8. Wing MR, Ziegler J, Langefeld CD, Ng MC, Haffner SM, Norris JM, et al. Analysis of FTO gene variants with measures of obesity and glucose homeostasis in the IRAS family study. Hum Genet. 2009;125:615-26.

9. Doney AS, Dannfald J, Kimber CH, Donnelly LA, Pearson E, Morris AD, et al. The FTO gene is associated with an atherogenic lipid profile and myocardial infarction in patients with type 2 diabetes: a genetics of diabetes audit and research study in Tayside Scotland (go-DARTS) study. Circ Cardiovasc Genet. 2009;2:255-9.

10. Raza ST, Abbas S, Siddiqi Z, Mahdi F. Association between ACE (rs4646994), FABP2 (rs1799883), MTHFR (rs1801133), FTO (rs9939609) genes polymorphism and type 2 diabetes with dyslipidemia. Int J Mol Cell Med. 2017;6:121-30

11. Kamura Y, Iwata M, Maeda S, Shinmura S, Koshimizu Y, Honoki H, et al. FTO gene polymorphism is associated with type 2 diabetes through its effect on increasing the maximum BMI in Japanese men. PLoS One. 2016;11: e0165523.

12. Ghafarian-Alipour F, Ziaee S, Ashoori MR, Zakeri MS, Boroumand MA, Aghamohammadzadeh N, et al. Association between FTO gene polymorphisms and type 2 diabetes mellitus, serum levels of apelin and androgen hormones among Iranian obese women. Gene. 2018;641:361-6.

13. Yang Y, Liu B, Xia W, Yan J, Liu HY, Hu L, et al. FTO genotype and type 2 diabetes mellitus: spatial analysis and meta-analysis of 62 case-control studies from different regions. Genes (Basel). 2017; 8(2):70.

14. Abu-Rmeileh NM, Husseini A, Capewell S, O'Flaherty M. Project M. Preventing type 2 diabetes among Palestinians: comparing five future policy scenarios. BMJ Open. 2013;3:e003558.

15. Abu-Rmeileh NM, Husseini A, O'Flaherty M, Shoaibi A, Capewell S. Forecasting prevalence of type 2 diabetes mellitus in Palestinians to 2030: validation of a predictive model. Lancet. 2012;380:S21.

16. El Sharif N, Samara I, Titi I, Awartani A. Compliance with and knowledge about diabetes guidelines among physicians and nurses in Palestine/ Connaissances et respect des recommandations Sur le diabete chez des medecins et des infirmieres en Palestine. East Mediterr Health J. 2015;21:791.

17. Damiri B, Aghbar A, Alkhdour S, Arafat Y. Characterization and prevalence of metabolic syndrome among overweight and obese young Palestinian students at an-Najah National University. Diabetes Metab Syndr. 2018; 12(3):343-8.

18. Ereqat $\mathrm{S}$, Nasereddin A, Azmi K, Abdeen Z, Amin R. Impact of the Pro12Ala polymorphism of the PPAR-Gamma 2 gene on metabolic and clinical characteristics in the Palestinian type 2 diabetic patients. PPAR Res. 2009; 2009:874126.

19. Ereqat S, Nasereddin A, Cauchi S, Azmi K, Abdeen Z, Amin R. Association of a common variant in TCF7L2 gene with type 2 diabetes mellitus in the Palestinian population. Acta Diabetol. 2010;47(Suppl 1):195-8.

20. Lopez-Bermejo A, Petry CJ, Diaz M, Sebastiani G, de Zegher F, Dunger DB, et al. The association between the FTO gene and fat mass in humans develops by the postnatal age of two weeks. J Clin Endocrinol Metab. 2008; 93:1501-5.

21. Grant SF, Li M, Bradfield JP, Kim CE, Annaiah K, Santa E, et al. Association analysis of the FTO gene with obesity in children of Caucasian and African ancestry reveals a common tagging SNP. PLoS One. 2008;3:e1746.

22. Price RA, Li WD, Zhao H. FTO gene SNPS associated with extreme obesity in cases, controls and extremely discordant sister pairs. BMC Med Genet. 2008;9:4

23. Bressler J, Kao WH, Pankow JS, Boerwinkle E. Risk of type 2 diabetes and obesity is differentially associated with variation in FTO in whites and African-Americans in the ARIC study. PLoS One. 2010;5:e10521.
24. Vasan SK, Karpe F, Gu HF, Brismar K, Fall CH, Ingelsson E, et al. FTO genetic variants and risk of obesity and type 2 diabetes: a meta-analysis of 28,394 Indians. Obesity (Silver Spring). 2014;22:964-70.

25. Al-Serri A, Al-Bustan SA, Kamkar M, Thomas D, Alsmadi O, Al-Temaimi R, et al.. Association of FTO rs9939609 with obesity in the Kuwaiti population: a public health concern? Med Princ Pract. 2018; 27(2):145-51.

26. Yajnik CS, Janipalli CS, Bhaskar S, Kulkarni SR, Freathy RM, Prakash S, et al. FTO gene variants are strongly associated with type 2 diabetes in south Asian Indians. Diabetologia. 2009;52:247-52

27. Chauhan G, Tabassum R, Mahajan A, Dwivedi OP, Mahendran Y, Kaur I, et al. Common variants of FTO and the risk of obesity and type 2 diabetes in Indians. J Hum Genet. 2011;56:720-6.

28. Merritt DC, Jamnik J, El-Sohemy A. FTO genotype, dietary protein intake, and body weight in a multiethnic population of young adults: a crosssectional study. Genes Nutr. 2018;13:4.

29. Saldana-Alvarez Y, Salas-Martinez MG, Garcia-Ortiz H, Luckie-Duque A, Garcia-Cardenas G, Vicenteno-Ayala H, et al. Gender-dependent association of FTO polymorphisms with body mass index in Mexicans. PLoS One. 2016; 11:e0145984.

30. Sentinelli F, Incani M, Coccia F, Capoccia D, Cambuli VM, Romeo S, et al. Association of FTO polymorphisms with early age of obesity in obese Italian subjects. Exp Diabetes Res. 2012;2012:872176.

31. Tan $\sqcup$, Zhu H, He H, Wu KH, Li J, Chen XD, et al. Replication of 6 obesity genes in a meta-analysis of genome-wide association studies from diverse ancestries. PLoS One. 2014;9:e96149.

32. Hubacek JA, Dlouha D, Klementova M, Lanska V, Neskudla T, Pelikanova T. The FTO variant is associated with chronic complications of diabetes mellitus in Czech population. Gene. 2018;642:220-4.

33. Wang Q, Wang J, Lin H, Huo X, Zhu Q, Zhang M. Relationship between fat mass and obesity-associated gene expression and type 2 diabetes mellitus severity. Exp Ther Med. 2018;15:2917-21.

34. Abdelmajed SS, Youssef M, Zaki ME, Hassan NA-M, Ismail S. Association analysis of FTO gene polymorphisms and obesity risk among Egyptian children and adolescents. Genes Dis. 2017;4:170-5.

35. Celis-Morales C, Marsaux CF, Livingstone KM, Navas-Carretero S, SanCristobal R, O'Donovan CB, et al. Physical activity attenuates the effect of the FTO genotype on obesity traits in European adults: the Food4Me study. Obesity (Silver Spring). 2016;24:962-9.

36. Khan SM, El Hajj Chehadeh S, Abdulrahman M, Osman W, Al SH. Establishing a genetic link between FTO and VDR gene polymorphisms and obesity in the Emirati population. BMC Med Genet. 2018;19:11.

37. Liu G, Zhu H, Lagou V, Gutin B, Stallmann-Jorgensen IS, Treiber FA, et al. FTO variant rs9939609 is associated with body mass index and waist circumference, but not with energy intake or physical activity in Europeanand African-American youth. BMC Med Genet. 2010;11:57.

38. Vasan SK, Fall T, Neville MJ, Antonisamy B, Fall CH, Geethanjali FS, et al. Associations of variants in FTO and near MC4R with obesity traits in south Asian Indians. Obesity (Silver Spring). 2012;20:2268-77.

39. Montesanto A, Bonfigli AR, Crocco P, Garagnani P, De Luca M, Boemi M, et al. Genes associated with type 2 diabetes and vascular complications. Aging (Albany NY). 2018;10:178-96

40. Liu C, Mou S, Pan C. The FTO gene rs9939609 polymorphism predicts risk of cardiovascular disease: a systematic review and meta-analysis. PLoS One. 2013;8:e71901.

Ready to submit your research? Choose BMC and benefit from:

- fast, convenient online submission

- thorough peer review by experienced researchers in your field

- rapid publication on acceptance

- support for research data, including large and complex data types

- gold Open Access which fosters wider collaboration and increased citations

- maximum visibility for your research: over $100 \mathrm{M}$ website views per year

At $\mathrm{BMC}$, research is always in progress.

Learn more biomedcentral.com/submission 